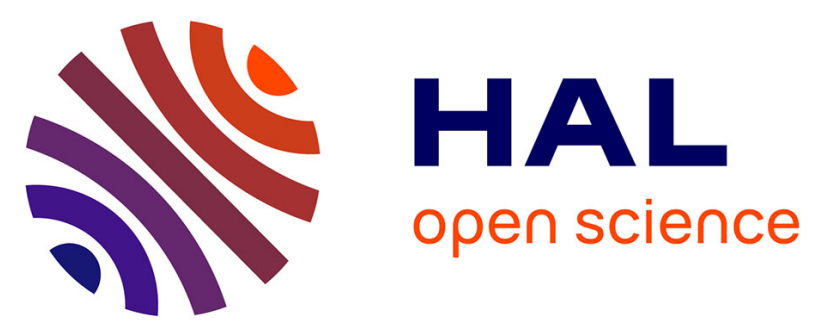

\title{
Defect-Driven Magnetization Configuration of Isolated Linear Assemblies of Iron Oxide Nanoparticles
}

\author{
Mircea V Rastei, Véronique Pierron-bohnes, Delphine Toulemon, Corinne
}

Bouillet, Attila Kakay, Riccardo Hertel, Ebenezer Tetsi, Sylvie Begin-Colin, Benoit P Pichon

\section{To cite this version:}

Mircea V Rastei, Véronique Pierron-bohnes, Delphine Toulemon, Corinne Bouillet, Attila Kakay, et al.. Defect-Driven Magnetization Configuration of Isolated Linear Assemblies of Iron Oxide Nanoparticles. Advanced Functional Materials, 2019, 29 (45), pp.1903927. 10.1002/adfm.201903927 . hal-02351831

\section{HAL Id: hal-02351831 \\ https://hal.science/hal-02351831}

Submitted on 6 Nov 2019

HAL is a multi-disciplinary open access archive for the deposit and dissemination of scientific research documents, whether they are published or not. The documents may come from teaching and research institutions in France or abroad, or from public or private research centers.
L'archive ouverte pluridisciplinaire HAL, est destinée au dépôt et à la diffusion de documents scientifiques de niveau recherche, publiés ou non, émanant des établissements d'enseignement et de recherche français ou étrangers, des laboratoires publics ou privés. 


\title{
Defect-Driven Magnetization Configuration of Isolated Linear Assemblies of Iron Oxide Nanoparticles
}

\author{
Mircea V. Rastei, ${ }^{1}$ Véronique Pierron-Bohnes, ${ }^{1}$ Delphine Toulemon, ${ }^{1}$ Corinne Bouillet, ${ }^{1}$ Attila Kakay ${ }^{2}$, Riccardo \\ Hertel, ${ }^{1}$ Ebenezer Tetsi, ${ }^{1}$ Sylvie Begin-Colin, ${ }^{1}$ Benoit P. Pichon ${ }^{*}, 1,3$
}

1 Université de Strasbourg, CNRS, Institut de Physique et Chimie des Matériaux de Strasbourg, UMR 7504, 23 rue du Loess BP 43, 67034 Strasbourg, Cedex 2, France

2 Helmholtz- Zentrum Dresden - Rossendorf, Dresden 01328, Germany

3 Institut Universitaire de France, 1 rue Descartes, 75231 Paris Cedex 05, France

\section{Benoit.Pichon@unistra.fr}

\section{Keywords}

One-NP- wide chains, magnetization states, AFM / MFM, e-holography, micromagnetic simulations, HR-TEM

\begin{abstract}
The magnetization state of one-dimensional magnetic nanoparticle chains plays a key role for a wide range of applications ranging from diagnosis and therapy in medicine to actuators, sensors and quantum recording media. The interplay between the exact particle orientation and the magnetic anisotropy is in turn crucial for controlling the overall magnetization state with high precision. Here, we report on a three-dimensional description of the magnetic structure of one-NP-wide chains. In this aim, we combined two complementary experimental techniques, magnetic force microscopy (MFM) and electronic holography $(\mathrm{EH})$ which are sensitive to out-of-plane and in-plane magnetization components, respectively. We extended our approach to micromagnetic simulations which provided results in good agreement with MFM and $\mathrm{EH}$. The findings are at variance with the known results on unidirectional nanoparticle assemblies, and show that magnetization is rarely strictly collinear to the chain axis. The magnetic structure of one-NP-wide chains can be interpreted as head-to-head magnetic domain structures with off-axis magnetization components, which is very sensitive to morphological defects in the chain structure such as minute size variation of NPs, tiny misalignment of NPs and/or crystal orientation with respect to easy magnetization axis.
\end{abstract}




\section{Introduction}

Unidirectional assemblies of nanoparticles (NPs) are driving a tremendous interest for a wide range of target applications ranging from diagnosis and therapy in medicine to actuators, sensors and quantum recording media. ${ }^{[1,2],[3]}$ Indeed, highly anisotropic assemblies of nanoparticles are likely to play a significant role in the improvement of the efficiencies of devices. The low dimensionality of nanoparticle chains enhances magnetic anisotropy which favors specific properties such as heat generation efficiency in magnetic hyperthermia. ${ }^{[4,5]}$ It is well known that the magnetic properties of nanoparticles are directly dependent of the interplay between magnetic anisotropy and dipolar interaction energies. ${ }^{[6]}$ Each nanoparticle behaves as a magnet so that their spatial arrangement is key point in mastering their magnetic properties. Dipolar interactions are dramatically enhanced when reducing the distance between nanoparticles. However, the magnetic properties of nanoparticles being usually studied as powders which can be assimilated to 3D random assemblies, the shape of nanoparticles (usually OD spherical or cubic) results in isotropic interactions which occur in nonselective direction, thus impeding the efficiency of the collective properties. Beside the OD morphology of nanoparticles, it is possible to induce shape anisotropy by controlling carefully the formation of nanoparticle assemblies. ${ }^{[7]}$ An attractive approach is to reduce the dimensionality of assemblies to control dipolar interactions in specific directions. For instance, 2D assemblies of nanoparticles were reported to exhibit enhanced magnetic anisotropy due to the selective orientation of magnetic moments in the plane of the monolayer ${ }^{[8-10]}$ Such a behavior was also demonstrated in multilayer assemblies by separating specifically each layer which were coupled in the antiferromagnetic configuration. ${ }^{[11]}$ Another study showed that the local anisotropy resulting from chain-like assemblies as pieces of a $2 \mathrm{D}$ monolayer enhanced markedly the magnetic anisotropy of nanoparticles. ${ }^{[12]}$

Therefore, enhancing shape anisotropy by decreasing the dimensionality to 1D chains results in much simpler magnetic anisotropic structures which enhanced collective properties. ${ }^{[13-16]}$ Magnetic nanoparticles assembled as chains are usually believed to consist in collinear magnetic moments along the chain axis. ${ }^{[6]}$ However, such ideal picture of the magnetic structure of unidirectional assemblies has not been directly observed yet. Indeed, the interplay between the intrinsic magnetic uniaxial anisotropy within NPs - which is related to the internal crystal structure and the precise configuration of the NPs in the chain - and the magnetic coupling arising within a linear configuration directly asks for local studies conducted on individual chains. For instance, helical superstructures of nanocubes driven by the interplay of weak and magnetic interactions were reported. ${ }^{[17]}$ NPs incorporated in ant body parts also revealed the effective deviation of the easy magnetization axis with the unidirectional assembly. ${ }^{[18]}$

However, the deep understanding of the magnetic structure and collective properties of highly anisotropic assemblies is hampered by the production of real 1D assemblies such as one-NP-wide chains. To date, individual linear chain-like arrangements have essentially been investigated into magnetotactic bacteria, ${ }^{[19],[20-22]}$ Therefore, the preparation of the NP unidirectional assemblies (i.e. density of chains and their length) is challenging because it depends on several parameters such as the intrinsic magnetic properties of NPs, chemical interaction between ligands at both substrate and NP surfaces, kinetics of assembly reaction, and gradient orientation and strength of the magnetic field. Recently, we developed a preparation technique for one-NP-wide chains consisting in applying a magnetic field gradient while performing the assembly of the NPs on a surface through selective chemical interactions by using click chemistry. ${ }^{[23]}$ The method is able to produce individual chains of NPs with a well-defined structure and spatial arrangement. Such one-dimensional chains became fascinating structures for investigations of local magnetization states. Beside demonstrating that the collective properties of single NP chains significantly differ from those of $2 \mathrm{D}$ monolayers or isolated 
nanoparticles, we showed that the magnetization of each nanoparticles was not collinear to the chain axis. It was ascribed to the interplay of dipolar interactions between NPs and between nanoparticle chains. However, it is now obvious that for sufficiently near chains there exists a first order intra-chain magnetic interaction and a second order inter-chain magnetic coupling. ${ }^{[23]}$ The magnetic configuration of isolated 1D chains have been scarcely investigated. Mostly, it was deduced from the easy magnetization axis determined by high resolution transmission electron microscopy (HR-TEM), ${ }^{[22]}$ but never directly by accurate magnetometry techniques.

Herein, we present a study which focuses on the magnetization states of iron-oxide NP chains of widths corresponding to a single NP size. This strategy allows us to focus on the intra-chain interactions in isolated chains and reveals the dominant role played by minute deviations from an ideal linear configuration. The originality of our approach is that the magnetic structure of the same NP chain was experimentally investigated in three dimension by magnetic force microscopy (MFM) and electronic holography $(\mathrm{EH})$. Furthermore, these experimental results were compared with micromagnetic simulations which precisely consider the shape and the crystal orientation; magnetization easy directions of each NP were determined by HR-TEM. 


\section{Results and discussion}

NPs which are considered here, were synthesized by the thermal decomposition method which allow the fine control of size distribution, shape and high colloidal stability in aprotic solvents thanks to coating by oleic acid. ${ }^{[24,25]}$ They belong to magnetite $\left(\mathrm{Fe}_{3} \mathrm{O}_{4}\right)$ although their surface is partially oxidized in maghemite $\left(\gamma-\mathrm{Fe}_{2} \mathrm{O}_{3}\right)$, as usually reported. ${ }^{[24]}$ They exhibit a narrow size distribution centered to $19.5 \pm 1.8 \mathrm{~nm}$ and can be considered as truncated octahedrons (Fig. SI-1). They were allowed to selfassemble into one-NP-wide chain upon solvent evaporation under a magnetic field of $300 \mathrm{mT}$ applied in the plane of a TEM grid after drop casting.

To analyze the magnetization configuration of individual NP chains, we performed a detailed highresolution atomic force microscopy study while concomitantly recording the topography using atomic force microscopy (AFM) and the magnetic stray field gradient using magnetic force microscopy (MFM). Figure 1a shows a large-scale AFM image of well separated one-NP-wide chains. The image shows a large amount of chains of various lengths and configurations with different particulate arrangements and morphologies. The corresponding MFM signal (Fig. 1b) reveals a first intriguing property, namely that the intrinsic magnetization configuration, in a large majority of chains, adopts a complex manifold magnetic structure relying on chain length and proximity effects. In particular, it is interesting to see that the longest and regular chains show an elevated magnetic signal, in contrast with broken chains or individual NPs were the magnetic signal is either weak or absent. Furthermore, a closer look reveals a segment-like magnetic pattern with a mean domain size of about $125 \mathrm{~nm}$. This value is first obtained from 2D- fast Fourier transform (FFT) maps of MFM images. As an example, the inset in Fig. 1c shows an FFT map of the MFM image from Fig. 1b. It discloses two equidistant lobes (white arrows) pointing as expected along the chains' axes. A line spectrum crossing the two lobes is shown in Fig. 1c, where the black arrows above the shoulders provide the smallest size of the magnetic structures. This information is also seen directly in MFM images, independently on lift heights, when imaging a particular chain (Fig. 1d). It is worth noting that such a three-dimensional magnetization structure presenting magnetic-like domains separated by abrupt domain walls is unexpected and remained unpredicted in isolated one-dimensional NP chains.

a

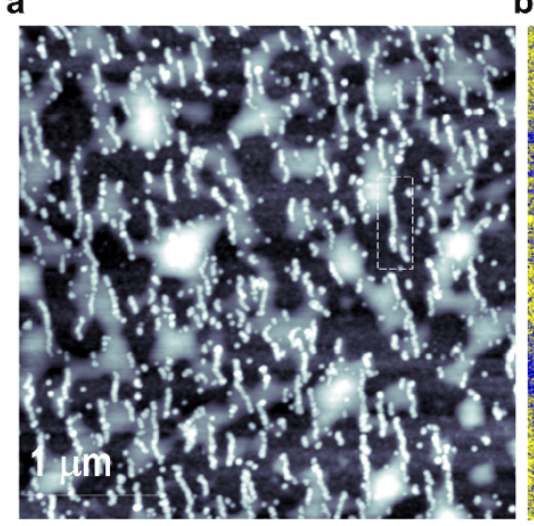

b

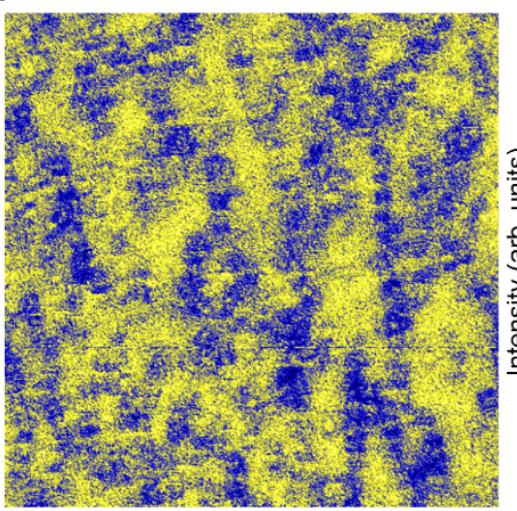

c

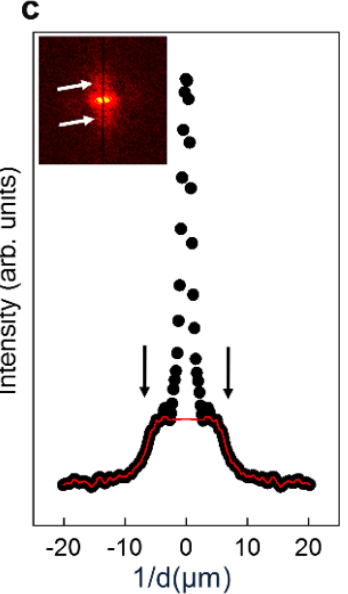

d

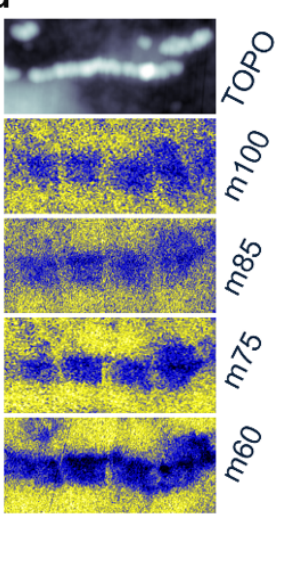

Figure 1. Magnetization imaging of nanoparticle chains. a, Nanoparticle chains casted on a silica substrate and imaged by atomic force microscopy. b. Magnetic response of the same area, obtained by magnetic force microscopy in phase contrast mode. c, Fast Fourier transform profile along the direction joining the spots marked by arrows in the inset. Black arrows indicate the same spectral positions as white arrows in the inset. Red curve is a smooth line through the data while neglecting the central peak. Inset: Full 2D Fast Fourier transform image of the magnetic signal mapped in d. Highresolution topography and corresponding magnetic images for the chain enclosed by the white rectangle in $\mathbf{a}$. The side labeling indicates the heights above the chain (in $\mathrm{nm}$ ) at which the magnetic signal has been measured. 
These global observations led us to prepare additional samples which consist in well separated chains in order to perform a detailed study including MFM, EH and HR-TEM. From the practical point of view, EH was performed first to avoid any exposition to magnetic field generated by the MFM tip. Figure 2(a) shows an AFM image of an area comprising three NP chains which were labelled as N4, N8 and $\mathrm{N} 9$ corresponding to the numbers of NPs. At the beginning of the EH study, each chain was imaged by TEM in order to reveal the exact morphology and NP number (Fig. 2b for N9). Fig. 2c-e show individual AFM images of the same chains. The corresponding MFM images are shown in Figs. 2f-k. These images show that N8 and N9 chains display a significant variation of the magnetic signal in the middle (Fig. 2l) which is ascribed to a domain-like magnetic structure and may originate from some deviation of NP sizes (Fig. 2b and SI-3). Nevertheless, the exact orientation of the magnetic moments is difficult to deduce at this stage. It is worth noting that the variation of the signal cannot be ascribed to scanning effect (Fig. SI-7). Anyway, a magnetic configuration comprising domains was not expected for such linear NP assemblies, as commonly admitted. ${ }^{[26]}$ A magnetic configuration with all NP magnetic moments aligned along the chain axis is usually observed as in magnetotactic bacteria. ${ }^{[21]}$ The size of the magnetic domains seems to be of about $4-5$ nanoparticles, i.e. a length of $80-100 \mathrm{~nm}$. These mean values are slightly smaller than the periodicity calculated in Fig. 1 and can be correlated to the length of NP chains. In contrast, the homogenous signal recorded for a smaller chain of 4 NPs (Fig. 2h) agrees with the absence of a domain structure. In our case, these results are indicative of the existence of a minimum number of NPs required to constitute a unique domain structure.
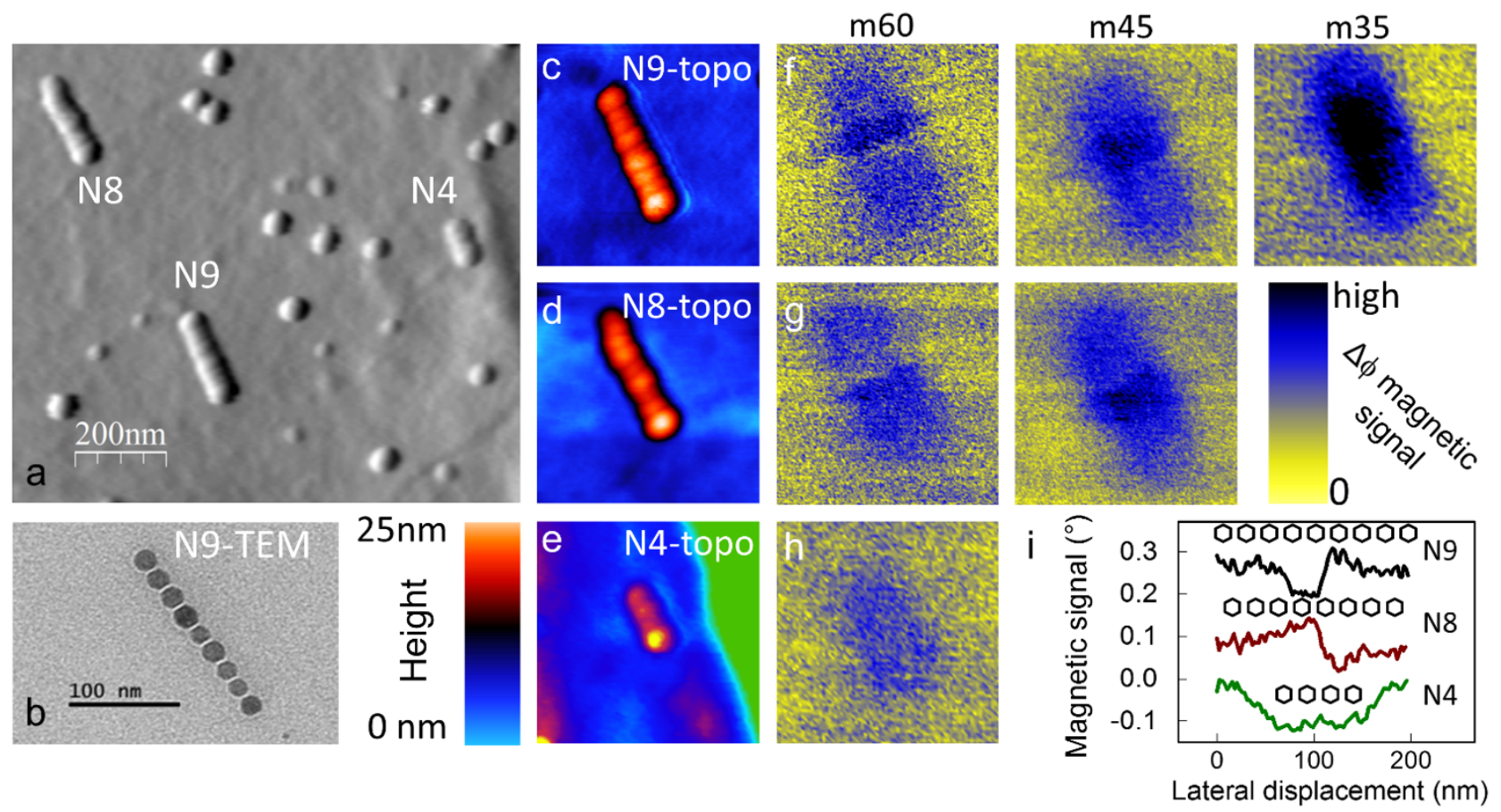

Figure 2 Effect of chain length on magnetization states. a, Large AFM topography of three non-interacting nanoparticle chains containing eight (N8), nine (N9), and four (N4) nanoparticles. b. Transmission electron microscopy of $\mathrm{N} 9$ chain with a small defocus to visualize the NPs. The image shows an example on how information about size distribution, arrangement and particle faceting within the chains is obtained (also see SI). c - e, AFM topographies of N8, N9, and N4 chains. $\mathbf{f}-\mathbf{k}$, Magnetic signal at various heights (in $\mathrm{nm}$ ) above the chains N8, N9, and N4. The heights are indicated on each image. I, Magnetic signal profiles at $60 \mathrm{~nm}$ height along the chains. The profiles were vertically shifted for sake of clarity. 
In order to understand the presence of such magnetic domais, we have investigated more deeply the chain structure (Fig. 3). AFM and TEM micrographs clearly show the presence of some defects such as NP of different sizes in chains N6 and N16, or additional NP located on the side of the N18 chain, indicated by white arrows in Fig. $3(a, f)$. The MFM images indeed revealed that the morphological defects represent specific sites where the magnetization is inhomogeneous, generating higher stray field gradients. An interesting aspect is that magnetic contrast instabilities induced by line scanning can be observed, as labeled by (1) in Fig. 3h. These are transitory instabilities of domain walls induced by the magnetic field of the tip, effect that can be eventually used to control the magnetization characteristics in devices based on magnetization propagation. ${ }^{[27-29]}$ Such instabilities are the signature of a domain wall jumping between metastable pinning positions, in a similar way as observed in magnetic continuous metallic structures. ${ }^{[30,31]}$

It is also interesting to remark that all isolated NPs do not show any magnetic signal in MFM images, which is likely due to their superparamagnetic states at room temperature. ${ }^{[32],[23]}$ Moreover, this absence of MFM signal above individual NPs also demonstrates that the stray field generated by the probes is not sufficient to keep the magnetization of these NPs in a fixed position, i.e. the energy barrier for flipping their magnetization is smaller than the magnetostatic energy provided by the tipNP interaction. According to the MFM observations mentioned above, it is clear that morphological defects into the chain structure induce inhomogeneities in magnetization and domain wall-like pinning sites. However, because of the probe oscillation geometry, MFM signal is highly sensitive to out-ofplane magnetic stray fields only, ${ }^{[33-35]}$ making difficult the prediction of a full 3D magnetization configuration. 
a

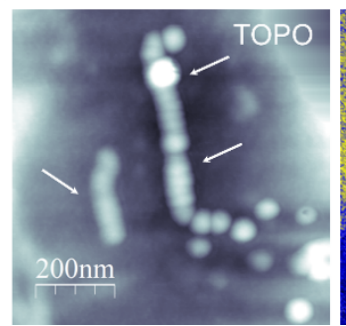

b
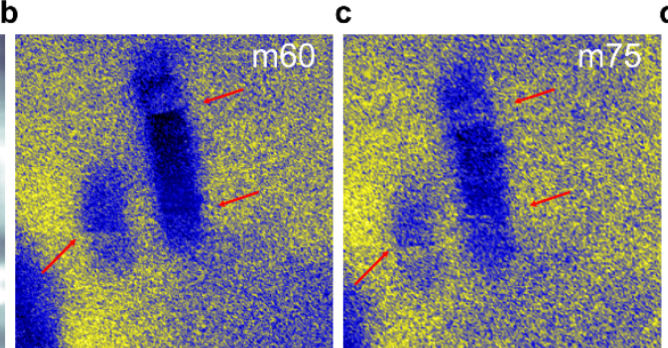

d
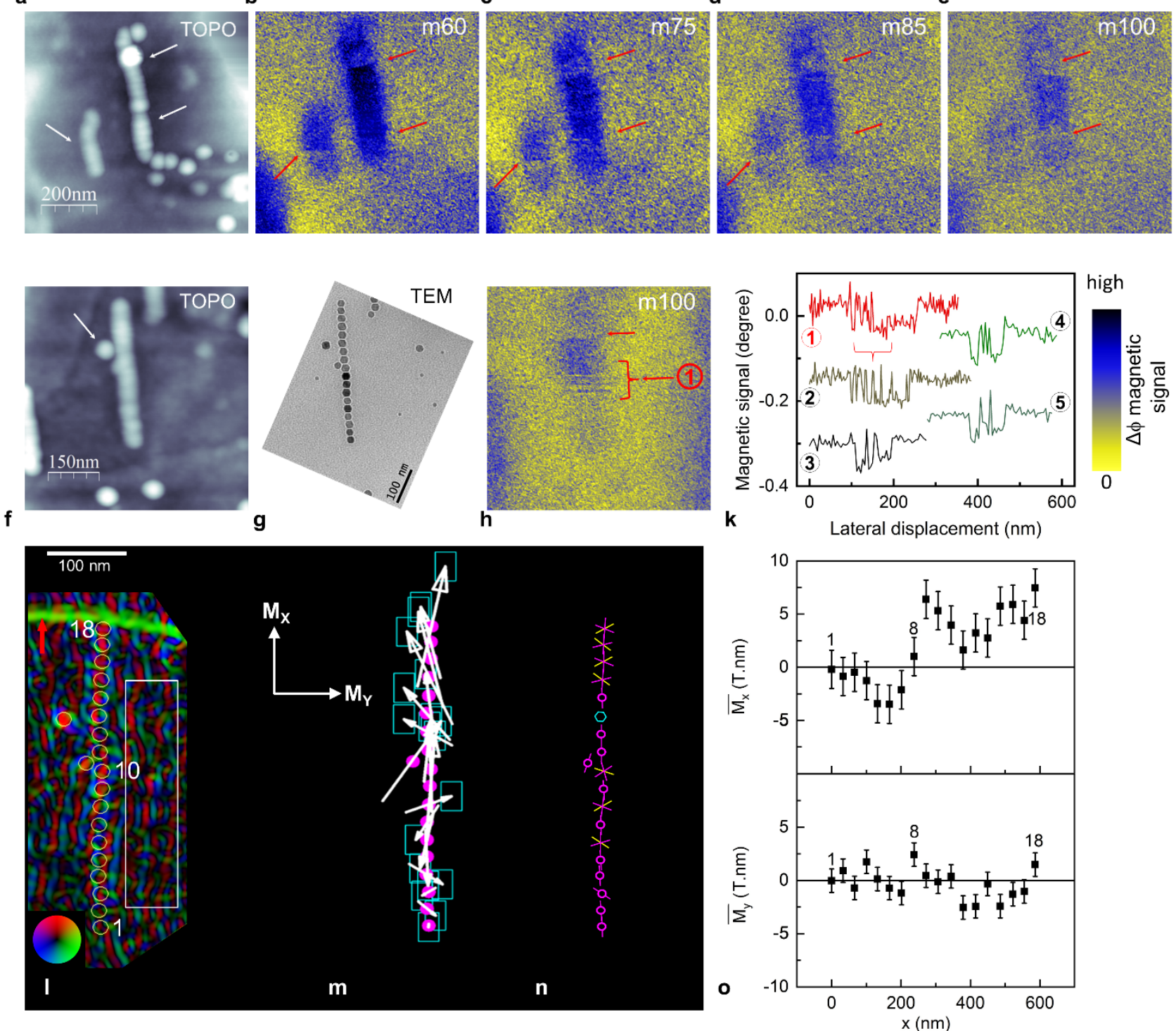

Figure 3 Role of the defects. a, AFM topography of two chains containing 6 (N6) and 16 (N16) nanoparticles, respectively. White arrows show local defects (NP of different size or additional NP). b-e, Corresponding MFM magnetic images acquired at various heights. The heights (in $\mathrm{nm}$ ) are indicated on each image. Red arrows point out domain transitions in the proximity of defects. f, AFM topography of a chain containing 18 nanoparticles (N18). g, TEM image of $\mathrm{N} 18$ chain. $\mathbf{h}$, Magnetic image of N18 at 100-nm height revealing domain wall-like instabilities (contrasts parallel to the scanning direction: the horizontal border of the image). $\mathbf{k}$, Examples of magnetic profiles along instable domain walls. Profiles (1) and (2) are acquired along the same domain wall marked by the bracket in $\mathbf{h}$, in two different images taken in the same conditions. Profiles (3) to (5) correspond to the domain wall shown by green arrows in Fig. SI-7 from supplementary materials. I-o, Magnetic configuration of NP18 chain calculated from holograms. I, Vector map of the in-plane components of the magnetic induction (the direction of the measured magnetic induction in the NPs is shown by a color wheel with a 15 T.nm integrated magnetization at the outer edge). The white rectangle indicates the range used to calculate the background and the error bar on the magnetization (average and standard deviation of the magnetic fluctuations). $\mathrm{m}$, Schematic moments of the NPs. The pink bullets show the particles positions and the black arrows show the orientation and amplitude of the moments. The red rectangles show the error bars on the $\overline{\mathrm{M}}_{x}$ and $\overline{\mathrm{M}}_{y}$ components, plotted in $\mathbf{o}$ as a function of the position, starting from the bottom. The $x$ coordinate is along the chain axis and $y$ coordinate is perpendicular to the chain axis in the TEM image plane. $\mathbf{n}$, Crystallographic orientation of the NPs: the direction normal to the membrane plane are [11-2] (circle), [110] (star), or [111] (hexagon). The magenta and yellow segments show the [111] and [001] in-plane directions, respectively. 
In order to gain more insights into the exact magnetic configuration of NP chains, we also performed electron holography $(\mathrm{EH})$ which is a powerful technique to investigate the magnetic structure of nanoobjects and their assemblies. ${ }^{[36-41],[42]} \mathrm{EH}$ is sensitive to in-plane magnetic induction and represents, in the frame of our study, an ideal tool to combine with MFM in order to gain a full picture of magnetic moment directions, i.e. a 3D magnetic qualitative information. Indeed, the differentiation of the magnetic part of the phase shift of the electron beam gives access to $\bar{M}$, the magnetization integrated along the electron trajectory (Figure 3o). The absolute magnetic moment of each NP is calculated by integrating $\bar{M}$ on its projected surface on the plane. The in-plane magnetic moments of the NPs are presented in Figure $3 \mathrm{~m}$ (see SI for details of analysis and more images).

In all chains, the magnetic moments of NPs are not strictly parallel to the chain axis and deviates from the usually assumed model. ${ }^{[43],[26]}$ In chain N9, $\overline{\mathbf{M}}_{x}$ and $\overline{\mathrm{M}}_{y}$ are rather constant which correspond to a ferromagnetic coupling of magnetic moments (see Fig. SI-14,15). Nevertheless, the NP \#1 exhibits a magnetic moment which is perpendicular to the chain axis $\left(\bar{M}_{x}=0\right)$ which agree with extremity effect. In addition, the magnetic moment of NP\#5 is almost parallel $\left(\overline{\mathrm{M}}_{y} \approx 0\right)$ which is correlated to the signal variation measured by MFM (Fig. 2f). Such a behavior confirms the role of defects such as NP size deviation (Fig. SI3c) in the formation of domain wall above a critical number of NPs. A longer chain such as NP18 shows significant variations of $\bar{M}_{x}$ while $\bar{M}_{y}$ remains close to zero (Fig. 3o). In this case, the sign change of $\overline{\mathbf{M}}_{x}$ corresponds to a domain wall. However, the decrease of $\overline{\mathbf{M}}_{x}$ is not compensated by the increase of $\overline{\mathrm{M}}_{y}$, which can be correlated to an out-of-plane magnetization component as observed in the MFM images (Fig. 3h). In addition, the presence of a supplementary NP on the chain side, nearby NP\#10 and NP\#11, seems to favor collinear magnetization to the chain axis (Fig. 3l,m). Their ferromagnetic coupling through dipolar interactions with a supplementary NP nearby the chain enhances locally magnetization as observed by the signal amplitude in this area measured by MFM (Fig. 3h). In contrast, a second NP, at about $40 \mathrm{~nm}$ from the chain, has no significant effect on magnetizations. The N30 chain also shows a domain-like structure; a domainwall is observed around NPs \#7-8. Moreover, the curved part above NP\#17 accounts from positioning defects which result in huge and irrational fluctuations of $\bar{M}_{x}$ and $\bar{M}_{y}$ (Fig. 4g,h and Fig. SI14b) among which antiferromagnetic coupling can be observed between NPs \#19-20 and \#28-29.

The magnetic properties of NP assemblies being directly related to the magnetocrystalline energy and dipolar energy, we investigated the orientation of the easy magnetization axis of each NP which correspond to the [111] crystallographic direction for magnetite. HRTEM micrographs successively recorded for 47 NPs of N18 (Fig. 3n) and N30 (Fig. 4i) chains showed that three different crystallographic orientations were observed in the direction parallel to the electron beam: [-211], [110], and [111] (Figure SI-8). We could assign the [-211] direction to 23 particles, the [1-10] direction to 19 particles, and the [111] direction to 5 particles which correspond to $49 \%, 40 \%$ and $11 \%$, respectively. Furthermore, 3D sketches that were calculated to correspond to 2D projections of NPs observed by TEM revealed that their shape is a truncated octahedron with large $\{111\}$ facets (Fig. SI8). NPs in the [-211] and [1-10] orientations, preferentially share $\{111\}$-type facets along the chain axis. This relative configuration is observed in 13 inter-particle planes over 17 in N18 and 16 over the 25 known in N30. As the easy magnetization axis of magnetite is perpendicular to these facets, the [211] and [1-10] orientations favor the coupling of magnetic moments of most of NP through dipolar interactions. 
The larger proportions of the [-211] and [1-10] orientations than the [111] orientation can be also explained by the self-assembly mechanism which is driven by interparticle interactions. The [111] planes are the largest facets of octahedrons. Therefore, they favor stronger Van-der-Waals interactions between the alkylene chains of oleic acid molecules grafted at the NP surface. ${ }^{[15]}$ In contrast, NPs deposited on the TEM membrane on a $\{111\}$ face favors Van der Waals interactions with the C-membrane. Therefore, these NPs face their neighbors with a [110]-type edge, which hampers the dipolar interactions between magnetic moments of NPs. The fact that they are not numerous shows that the self-assembly of NPs is not driven by kinetic control, i.e. solvent evaporation. ${ }^{[44]}$ Otherwise, most of NPs would stand on their (111) face in order to optimize the interaction with the TEM membrane. Indeed, self-assembly mainly occurs in the solution.

Therefore, both magnetic and chemical interactions act concomitantly to drive the self-assembly of NPs with magnetic moments along the chain axis before the evaporation of the solvent was completed. ${ }^{[15]}$ However, it does not explain that magnetic moments are not aligned along the chain axis as it is usually reported. Indeed, the off-axis component of magnetic moments certainly accounts from the deviation of NP positions from the chain axis when chains enter in contact with the TEM membrane after evaporation. However, it does not result from the deposition of NPs on their larger $\{111\}$ facet on the membrane because dipolar interactions between NPs already promote the selfassembly process in solution. Furthermore, as we also mentioned above, the size variation of NPs which result in the variation of magnetization also plays a significant role. 


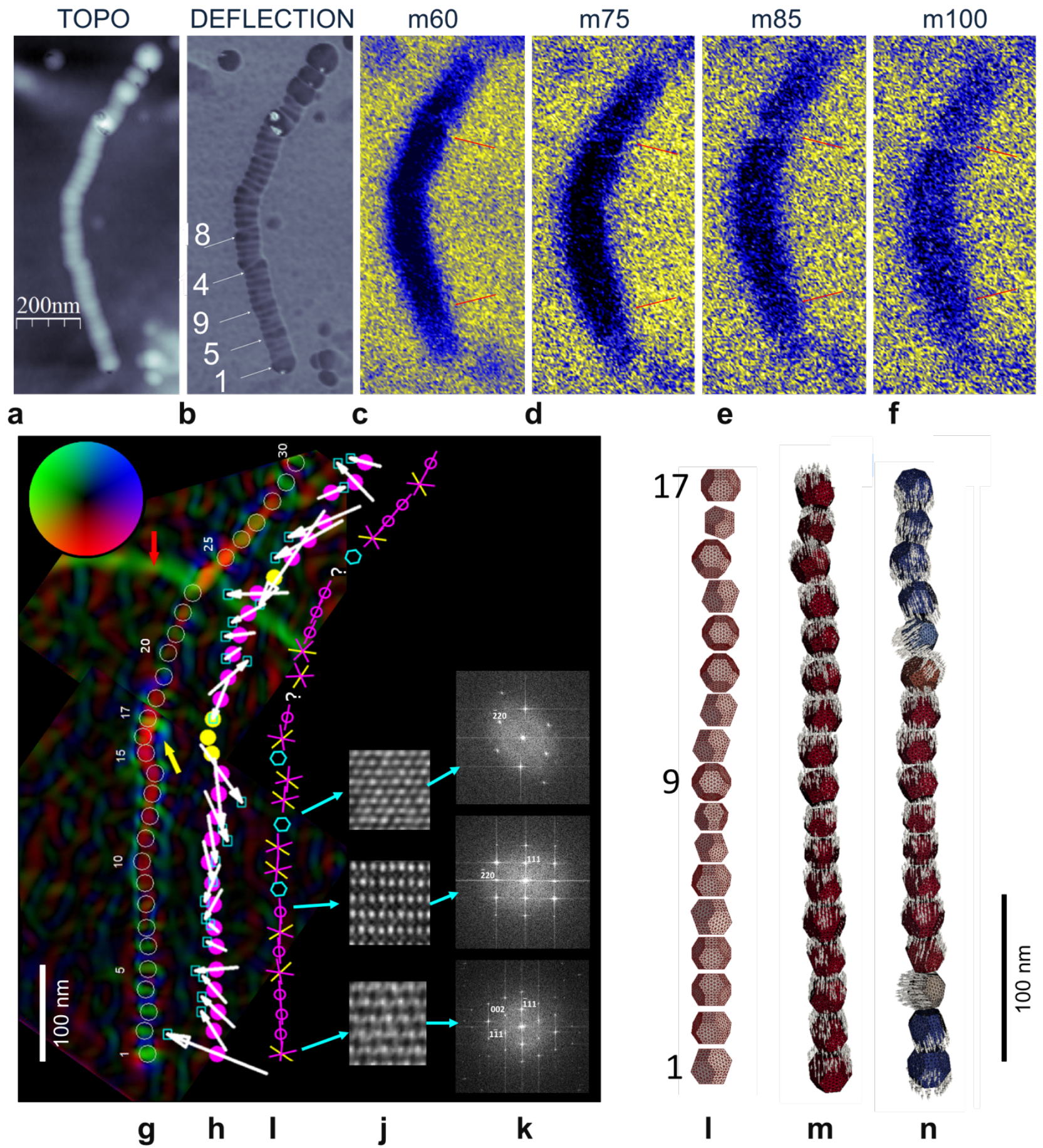

Figure 4. The case of long chains. a, Large AFM topography of N30, a long chain containing 30 nanoparticles. b, Deflection image revealing details on nanoparticles' number and arrangement (see also Fig. SI-3). c - $\mathbf{f}$, Magnetic signal at different distances (in $\mathrm{nm}$ ) above the chain. Red arrows indicate positions where the signal changes by more than $30 \%$. gh, Electronic holography performed on NP30. The different representations are shifted for clarity. g: Combination of $\overline{\mathbf{M}}_{x}$ and $\overline{\mathrm{M}}_{y}$ to visualize the integrated magnetization. The white circles show NPs' location. The yellow and red arrows show the location of experimental artefacts (isolated NP on the membrane in the reference beam and limit of membrane hole on the reference hologram, respectively). The color-scale circle shows the direction of the magnetization and corresponds to a 15 T.nm magnetization integrated on the beam trajectory at the outer edge. $\mathbf{h}$ : White arrows show the direction and amplitude of the in-plane magnetization $\overline{\mathrm{M}}$ for each NP which was integrated on the white circles. The rectangles show the 
error bars on both $\overline{\mathrm{M}}_{x}$ and $\overline{\mathrm{M}}_{y}$ components. The yellow bullets show the particle positions where it was not possible to deduce the magnetization direction due to artefacts. i: Crystallographic orientations of the NPs: the direction normal to the membrane plane are [11-2] (circle), [110] (star), or [111] (hexagon). The magenta and yellow segments show the [111] and [001] in-plane directions, respectively. I-n: Micromagnetic simulations. I: Finite-element model of an ensemble of 17 NPs corresponding to the \#1-\#17 segment of the N30 chain. n: A homogeneous magnetization state, with minor deviations of the magnetization from the chain axis. $m$ : The most inhomogeneous state containing three magnetic domains (e.g. two domain walls).

To assess the role of morphological defect in the chain of self-assembled $\mathrm{Fe}_{3} \mathrm{O}_{4} \mathrm{NPs}$, we conducted a systematic micromagnetic simulation study (Fig. 4I-n). By carefully analyzing the TEM micrographs corresponding the NPs \#1 to \#17 of the N30 chain, we first reproduced a three-dimensional model with the same shape, size, position, and crystal orientation of each NP (Fig. 4I and Fig. SI-5). The goal of preparing such a one-to-one correspondence between the experimentally observed structure and the simulation model is to improve the understanding of the interplay between the magnetization and the geometric assembly of the NPs. To simulate a situation corresponding to the experimental measurement, we assume an equilibrium state of the magnetization at zero field. In order to account for possible metastable states, we performed several tens of simulations with different, random initial starting configurations. ${ }^{[45]}$ If the simulations are started with a homogeneous initial magnetic configuration, they practically always converged towards a single-domain magnetization state, irrespective of the orientation of the initial magnetization, with the final magnetization aligned along the axis of symmetry (Fig. 4n). However, relaxing the magnetization at zero field starting from random initial configurations yields more frequently an inhomogeneous state, with two or sometimes three domains aligned along the axis of the chain (Fig. $4 \mathrm{~m}$ ). The separation between these head-to-head or tail-to-tail domains does not resemble to a usual domain wall. The transition consists mainly of a single NP with magnetization direction perpendicular to the axis of the chain, strongly misaligned with it, in agreement with electronic holography.

In a next step, we investigated the extent to which the occurrence of such domain states is due to a degree of structural disorder of the chain. For this purpose, we prepared a model consisting of another, similar chain of 17 nanoparticles (Fig. SI-5). This model serves as a comparison to the realistic chain with a larger degree of disorder. In contrast to the previously described chain (Fig. $4 \mathrm{~m}$ ), which tried to capture every geometric feature of inter-particle spacing, particle size and crystal orientation, this chain is "ideal" in the sense that it consists of perfectly aligned, equally spaced nanocrystals of identical size and orientation. The interparticle spacing and the particle size are such that the resulting chain allows for an optimal comparison between the ordered and the irregular model reconstructed from the experiment. We performed numerous simulations of the magnetic structure in the ordered chain in which different random magnetic structures were used as initial configurations. The results of these simulations yielded a correlation between the degree of order of the chain and the density of domain walls: The three-domain state was observed less frequently in the ordered chain than in the model of the real, imperfectly ordered chain. In total, we performed about 100 simulations to investigate the possible equilibrium structures of the magnetization in the ordered and in the realistic chain (Table 1). A significant increase of the two-domain state occurs in the realistic chain, indicating an increase of domain wall density with increasing disorder. These results corroborate the hypothesis that morphological defects play an important role in the occurrence of domains or domain walls in such chains of magnetic NPs. 
Table 1. Frequency of the resulting equilibrium magnetization states for the irregular chain and for the ideally ordered chain, starting from random initial configurations.

\begin{tabular}{|l|l|l|}
\hline & Irregular chain & Ordered chain \\
\hline Single-domain & $30 \%$ & $32 \%$ \\
\hline One domain wall & $47 \%$ & $57 \%$ \\
\hline Two domain walls & $23 \%$ & $10 \%$ \\
\hline
\end{tabular}

Remarkably, none of the simulations shows a state where the magnetization within a single NP is strongly inhomogeneous. This can be explained, of course, primarily by the small size of the NPs and therefore by the dominating influence of the exchange interactions between Fe cations. Nevertheless, a high-remanence state of individual NPs is also favored by the magnetostatic interaction, as it allows a favorable coupling to neighboring particles

The precise magnetic properties of the NPs are difficult to establish. A critical input parameter of the simulations is the value of magnetic anisotropy, which in such nanocrystals is likely to differ from the bulk value, for instance due to surface anisotropy. However, since the specific material parameters of the NPs are unknown, we used the bulk values: first cubic magnetocrystalline anisotropy constant $K_{\mathrm{c} 1}=-1.35 \cdot 10^{4} \mathrm{~J} / \mathrm{m}^{3}$, saturation magnetization $\mu_{0} M_{\mathrm{s}}=0.6 \mathrm{~T}\left(\mu_{0}\right.$ is the vacuum permeability), and exchange constant $A=13 \mathrm{pJ} / \mathrm{m}$.

To test the impact of the anisotropy, we performed a few additional simulations using the opposite sign of $\mathrm{K}$, in order to study a situation that differs diametrically from the previous calculations in terms of the magnetocrystalline anisotropy. To our surprise, the results of these simulations (not shown) do not display any qualitative difference compared to the other results. This indicates that the magnetostatic interaction between the NPs as well as the exchange interaction, which tends to preserve a homogeneous magnetization within each NP, are dominant for the result of the magnetic structure, i.e., that they play a far more important role than the magnetocrystalline anisotropy of the nanoparticles. These results agree with our previous study where we used the same code to highlight the impact of inter-particle magnetostatic coupling based on individual hysteresis loops of nanoparticles ${ }^{[46]}$ Similar observations were also reported for metallic nanostructures studied by MFM. [47-49] and $\mathrm{Fe}_{3} \mathrm{O}_{4} \mathrm{NP}$ chains biosynthesized by magnetotactic bacteria for which the dipolar energy was almost one order of magnitude higher than magnetocrystalline energy. ${ }^{[4]}$

\section{Conclusion}

The main objective of our study was to investigate the magnetic structure of individual NP chains in three-dimension and to correlate it to the spatial arrangement of each NP with respect to chain axis and crystal orientation. It is worth to note that one-NP-wide chains display permanent magnetization although isolated NPs of $20 \mathrm{~nm}$ are superparamagnetic. ${ }^{[23]} \mathrm{EH}$ and MFM being performed at room temperature with very small magnetic field $(<5 \mathrm{mT})$, it shows the dramatic effect of highly anisotropic assemblies on the magnetic anisotropy energy of NPs which is directly enhanced by dipolar interactions. 
Nevertheless, MFM and EH which are out-of-plane and in-plane magnetic sensitive techniques, respectively, unambiguously corroborated the existence of off-axis component of magnetization for NP chains of lengths from 8 to 30 NPs. As shown by the crystal orientation of each NP measured by HRTEM, out-of-plane and in-plane magnetization components could not result from the misalignment of easy magnetization axes of NPs. Indeed, they were ascribed to morphological defects relying on NP synthesis (NP size distribution) and self-assembly process (deviation of NP position from chainaxis or supplementary NP nearby a chain). Besides the systematic presence of off-axis magnetization components, magnetic domains were observed above a critical chain length of 8 NPs which agree with energy minimization of the system. Micromagnetic simulations confirmed that magnetic domains exist in order to minimize the magnetostatic energy. Unusual domain walls with head-to-head configuration (no antiferromagnetic coupling) could be perfectly observed by $\mathrm{EH}$ and micromagnetic simulations. Such a domain structure was correlated to an off-axis magnetization component resulting from defects in the chain structure.

In comparison to other studies on unidirectional assemblies which consist in bundled NP chains, magnetization of one-NP-wide chains is highly sensitive to morphology defects. Indeed, the presence of supplementary NP nearby the chain favored the local in-axis orientation of magnetization through collective dipolar interactions as usually reported at larger extent in bundled NP chains. ${ }^{[22]}$ Although dipolar energy $\left(E_{d}=1.610^{-10} \mathrm{erg}\right)$ is much larger than magnetic anisotropy energy $\left(E_{a}=1.9 .10^{-12} \mathrm{erg}\right)$, as confirmed by MFM measurements and micromagnetic simulations, such deviations of magnetic moments can only be ascribed to morphology defects which predominate in one-NP-wide chains. Our findings are highly decisive for the development of future devices based on NP chains for application fields such as magneto-resistive sensors and biomedicine. Indeed, tuning the arrangement of NPs with only small defects open possibilities toward manipulating the magnetic behavior of such chains.

\section{Acknowledgment.}

D.T. acknowledge Région Alsace and Direction Générale de l'Armement for financial support of her PhD thesis. This work was funded by the French National Research Agency (ANR) through the Programme d'Investissement d'Avenir under contract ANR-11-LABX-0058_NIE within the Investissement d'Avenir program ANR-10-IDEX-0002-02. Financial supports were also provided by the Agence Nationale pour la Recherche (ETNAA ANR-07-NANO-018) and METSA network for holography. V.P.B. and C.U. acknowledge Dr. Christophe Gatel and Dr. Aurélien Masseboeuf (CEMES Laboratory in Toulouse, France) for their kind help with the holography work.

Supporting information. Nanoparticles structural and magnetic characterizations. HRTEM micrographs and corresponding simulated sketches depicting crystal orientations, additional TEM and MFM micrographs, holography details, micromagnetic simulation images. 


\section{Materials and methods}

Synthesis of NP. Iron oxide NPs were synthesized as we reported previously. ${ }^{[12]}$ Iron stearate (1.38 $\mathrm{g}, 2.22 \mathrm{mmol}$ ) and oleic acid (1.24 g, $4.44 \mathrm{mmol})$ were added to $20 \mathrm{~mL}$ of docosene (b.p. $364{ }^{\circ} \mathrm{C}$ ). The mixture was first heated to $100{ }^{\circ} \mathrm{C}$ and kept under stirring for $1 \mathrm{~h}$. The reaction medium was subsequently heated under air atmosphere to reflux for $2 \mathrm{~h}$ (heating rate of $5{ }^{\circ} \mathrm{C} \mathrm{min}^{-1}$ ). The nanoparticles were then washed several times with a $\mathrm{CHCl}_{3} / \mathrm{EtOH}$ mixture by performing centrifugation in order to remove docosene, excess of oleic acid and side products. Finally, nanoparticles were obtained as a black and highly stable colloidal suspension after dispersion in THF.

Preparation of NP chains. Isolated NP chains with different length were prepared by controlling the slow evaporation of a highly diluted colloidal suspension after drop cast onto a TEM grid. A magnet placed below (magnetic field of $300 \mathrm{mT}$ ) the TEM grid before performing drop cast allowed to form NP chains upon THF evaporation at room temperature. The low concentration of NPs in the suspension allowed us to obtain chain lengths ranging from 4 to 30 nanoparticles.

Electron holography studies of the magnetic configuration of nanoparticle chains have been done by using an off-axis electron holography ${ }^{[50-52]}$ on an JEOL $2100 \mathrm{~F}$ microscope equipped with an electrostatic biprism. The sample containing these chains was not subject to any external field after the deposition on the TEM carbon membrane and before the end of the holography process. Therefore, these measurements were performed before MFM experiments in order to avoid any manipulation of NPs with the magnetic tip. The residual field of the objective lens has been compensated with an inverse current (field during hologram images smaller than $1 \mathrm{mT}$ ). The holograms have been registered with a $30-40 \mathrm{kX}$ magnification by applying a potential $(100-150 \mathrm{~V})$ to the biprism. The phase change of the electron wave due to the sample crossing is deduced using the GPA software. It contains an electric term due to the electron distribution and a magnetic term containing information on the magnetization distribution. It has been corrected from instrumental background by subtracting the phase of a reference hologram where both beams cross a hole or an empty membrane.

The chains, deposited on a labeled grid covered with a very thin carbon membrane ( $2 \mathrm{~nm}$ thick, prepared in the lab), were imaged both before and after reversal of the sample. The magnetic phase was deduced from the phase difference between both positions. The magnetic phase is related to the integral of the $z$-component of the magnetic potential $A_{z}$ along the electrons' path:

$\varphi_{\text {mag }}(x, y)=-\frac{e}{\hbar} \int A_{z}(x, y, z) d z$

The derivation of the magnetic phase provides the magnetization integrated along the electron beam trajectory (see SI for details) using:

$\left.\overline{\mathrm{M}}_{x}(x, y)=\int \mu_{o} M_{x}(x, y, z)\right) d z=\frac{\hbar}{e} \frac{\partial \varphi_{\text {mag }}(x, y)}{\partial y}$ and $\left.\overline{\mathrm{M}}_{y}=-\int \mu_{o} M_{y}(x, y, z)\right) d z=\frac{\hbar}{e} \frac{\partial \varphi_{m a g}(x, y)}{\partial x}$.

High resolution TEM micrographs were recorded with a 2100 JEOL microscope on chains deposited on a very thin carbon membrane for holography. This study was performed after the holography measurements because it induces a high field applied to the sample (around 2T).

MFM images were acquired with a Bruker AFM operating with a Nanoscope $V$ controller. All magnetic force microscopy (MFM) images were obtained in lift mode while working in air at atmospheric 
pressures. The topographic signals were recorded in soft tapping mode with probe amplitude oscillations around $15 \mathrm{~nm}$. We used cantilevers with a normal stiffness of the order of $3 \mathrm{~N} / \mathrm{m}$ resulting in a first normal-resonant mode of about $75 \mathrm{kHz}$. All AFM tips had apex radii around $5 \mathrm{~nm}$ with an upper conical shape of about $25^{\circ}$. These geometrical parameters kept to a minimum the tip convolution effects. Under these experimental conditions, all probes have provided equivalent results. The electrostatic force gradients due to tip-sample work function difference have been evaluated one order of magnitude smaller than the magnetostatic force gradient used for MFM images.

Micromagnetic simulations: Using a GPU-accelerated ${ }^{[53]}$ custom-developed finite-element algorithm (TetraMag ${ }^{[54]}$ ), we solve the Landau-Lifshitz-Gilbert equation ${ }^{[55,56]}$ for an ensemble of 17 magnetic nanoparticles corresponding to the \#1-\#17 segment of NP30 chain. The nanoparticles are magnetostatically coupled. The simulations are based on micromagnetic theory. ${ }^{[57,58]}$ Although the results show that the individual nanoparticles are sufficiently small to be considered as magnetic single-domain particles, ${ }^{[59]}$ we do not rely on any simplifying assumption regarding the magnetic structure such as, e.g., a macrospin model. To calculate the long-range magnetostatic interaction we employ a hybrid finite-element / boundary element algorithm, ${ }^{[60]}$ improved by a hierarchical matrix algorithm, ${ }^{[61]}$ which allows us to overcome the memory limits connected with classical BEM methods (see SI). We used truncated octahedra as a model for the geometric form of the nanoparticles. The discretization cells used in the simulations are significantly smaller than the nanoparticle itself, as each nanoparticle is subdivided into irregular tetrahedral finite elements with a size of about $1.2 \mathrm{~nm}$. The magnetization is calculated at the nodes of the cells, and it is interpolated linearly within each finite element. The magnetization is calculated at the nodes of the cells, and it is interpolated linearly within each finite element. We solve the Landau-Lifshitz-Gilbert equation at zero applied field to find stable configurations of the magnetization. We assume cubic anisotropy of the particles, with negative anisotropy constant. In each nanocrystal, the direction of the hard axes is along the six permutations of its (100) direction. 


\section{References}

[1] J. Yuan, Y. Xu, A. H. E. Müller, Chem. Soc. Rev. 2011, 40, 640.

[2] H. Kitching, M. J. Shiers, A. J. Kenyon, I. P. Parkin, J. Mater. Chem. A 2013, 1, 6985.

[3] H. U. I. Wang, Y. Yu, Y. Sun, Q. Chen, Nano 2011, 06, 1.

[4] C. Martinez-Boubeta, K. Simeonidis, A. Makridis, M. Angelakeris, O. Iglesias, P. Guardia, A. Cabot, L. Yedra, S. Estradé, F. Peiró, Z. Saghi, P. A. Midgley, I. Conde-Leborán, D. Serantes, D. Baldomir, Sci. Rep. 2013, 3, 1652.

[5] B. Mehdaoui, R. P. Tan, A. Meffre, J. Carrey, S. Lachaize, B. Chaudret, M. Respaud, Phys. Rev. B 2013, 87.

[6] S. Singamaneni, V. N. Bliznyuk, C. Binek, E. Y. Tsymbal, J. Mater. Chem. 2011, 21, 16819.

[7] Z. Tang, N. A. Kotov, Adv. Mater. 2005, 17, 951.

[8] P. Poddar, T. Telem-Shafir, T. Fried, G. Markovich, Phys. Rev. B 2002, 66, 060403.

[9] M. Pauly, B. P. Pichon, P. Panissod, S. Fleutot, P. Rodriguez, M. Drillon, S. Begin-Colin, J. Mater. Chem. 2012, 22, 6343.

[10] B. Faure, E. Wetterskog, K. Gunnarsson, E. Josten, R. P. Hermann, T. Bruckel, J. W. Andreasen, F. Meneau, M. Meyer, A. Lyubartsev, L. Bergstrom, G. Salazar-Alvarez, P. Svedlindh, Nanoscale 2013, 5, 953.

[11] B. P. Pichon, P. Louet, O. Felix, M. Drillon, S. Begin-Colin, G. Decher, Chem. Mater. 2011, 23 , 3668.

[12] D. Toulemon, Y. Liu, X. Cattoën, C. Leuvrey, S. Bégin-Colin, B. P. Pichon, Langmuir 2016, 32, 1621.

[13] Y. Zhang, L. Sun, Y. Fu, Z. C. Huang, X. J. Bai, Y. Zhai, J. Du, H. R. Zhai, J. Phys. Chem. C 2009, 113,8152 .

[14] C. Petit, V. Russier, M. P. Pileni, J. Phys. Chem. B 2003, 107, 10333.

[15] Y. Lalatonne, J. Richardi, M. P. Pileni, Nat. Mater. 2004, 3, 121.

[16] K. Nakata, Y. Hu, O. Uzun, O. Bakr, F. Stellacci, Adv. Mater. 2008, 20, 4294.

[17] G. Singh, H. Chan, A. Baskin, E. Gelman, N. Repnin, P. Král, R. Klajn, Science 2014, 345, 1149.

[18] O. C. Alves, R. B. Srygley, A. J. Riveros, M. A. Barbosa, D. M. S. Esquivel, E. Wajnberg, J. Phys. Appl. Phys. 2014, 47, 435401.

[19] R. E. Dunin-Borkowski, M. R. McCartney, R. B. Frankel, D. A. Bazylinski, M. Pósfai, P. R. Buseck, Science 1998, 282, 1868.

[20] M. Pósfai, T. Kasama, R. E. Dunin-Borkowski, In Magnetoreception and Magnetosomes in Bacteria; Schüler, D., Ed.; Springer Berlin Heidelberg, 2007; Vol. 3, pp. 197-225.

[21] R. B. Proksch, T. E. Schäffer, B. M. Moskowitz, E. D. Dahlberg, D. A. Bazylinski, R. B. Frankel, Appl. Phys. Lett. 1995, 66, 2582.

[22] E. Alphandery, Y. Ding, A. T. Ngo, Z. L. Wang, L. F. Wu, M. P. Pileni, ACS Nano 2009, 3, 1539.

[23] D. Toulemon, M. V. Rastei, D. Schmool, J. S. Garitaonandia, L. Lezama, X. Cattoën, S. BéginColin, B. P. Pichon, Adv. Funct. Mater. 2016, 26, 2454.

[24] A. Demortiere, P. Panissod, B. P. Pichon, G. Pourroy, D. Guillon, B. Donnio, S. Begin-Colin, Nanoscale 2011, 3, 225.

[25] W. Baaziz, B. P. Pichon, S. Fleutot, Y. Liu, C. Lefevre, J.-M. Greneche, M. Toumi, T. Mhiri, S. Begin-Colin, J. Phys. Chem. C 2014, 118, 3795.

[26] P. Panissod, M. Drillon, In Magnetism: Molecules to Materials IV; Miller, J. S.; Drillon, M., Eds.; Wiley VCH, 2003; pp. 233-270.

[27] Y. Nakatani, A. Thiaville, J. Miltat, Nat. Mater. 2003, 2, 521.

[28] J.-Y. Chauleau, R. Weil, A. Thiaville, J. Miltat, Phys. Rev. B 2010, 82, 214414.

[29] L. Thevenard, C. Gourdon, S. Haghgoo, J.-P. Adam, H. J. von Bardeleben, A. Lemaître, W. Schoch, A. Thiaville, Phys. Rev. B 2011, 83, 245211.

[30] X. Zhu, P. Grütter, V. Metlushko, B. Ilic, J. Appl. Phys. 2002, 91, 7340. 
[31] and P. Grutter, IEEE Trans. Magn. 2003, 39, 3420.

[32] S. Bedanta, W. Kleemann, J. Phys. Appl. Phys. 2009, 42, 013001.

[33] X. Zhu, P. Grütter, MRS Bull. 2004, 29, 457.

[34] M. V. Rastei, R. Meckenstock, J. P. Bucher, Appl. Phys. Lett. 2005, 87, 222505.

[35] M. Abes, M. V. Rastei, J. Venuat, A. Carvalho, S. Boukari, E. Beaurepaire, P. Panissod, A. Dinia, J. P. Bucher, V. Pierron-Bohnes, J. Appl. Phys. 2009, 105, 113916.

[36] E. Snoeck, C. Gatel, L. M. Lacroix, T. Blon, S. Lachaize, J. Carrey, M. Respaud, B. Chaudret, Nano Lett. 2008, 8, 4293.

[37] L.-M. Lacroix, S. Lachaize, F. Hue, C. Gatel, T. Blon, R. P. Tan, J. Carrey, B. Warot-Fonrose, B. Chaudret, Nano Lett. 2012, 12, 3245.

[38] C. Gatel, F. J. Bonilla, A. Meffre, E. Snoeck, B. Warot-Fonrose, B. Chaudret, L.-M. Lacroix, T. Blon, Nano Lett. 2015, 15, 6952.

[39] R. E. Dunin-Borkowski, T. Kasama, A. Wei, S. L. Tripp, M. J. Hÿtch, E. Snoeck, R. J. Harrison, A. Putnis, Microsc. Res. Tech. 2004, 64, 390.

[40] J. M. Thomas, E. T. Simpson, T. Kasama, R. E. Dunin-Borkowski, Acc. Chem. Res. 2008, 41, 665.

[41] V. Reichel, A. Kovács, M. Kumari, É. Bereczk-Tompa, E. Schneck, P. Diehle, M. Pósfai, A. M. Hirt, M. Duchamp, R. E. Dunin-Borkowski, D. Faivre, Sci. Rep. 2017, 7, 45484.

[42] B. P. Pichon, O. Gerber, C. Lefevre, I. Florea, S. Fleutot, W. Baaziz, M. Pauly, M. Ohlmann, C. Ulhaq, O. Ersen, V. Pierron-Bohnes, P. Panissod, M. Drillon, S. Begin-Colin, Chem Mater 2011, 23, 2886.

[43] M. Charilaou, J. Kind, I. García-Rubio, D. Schüler, A. U. Gehring, Appl. Phys. Lett. 2014, 104, 112406.

[44] Y. Min, M. Akbulut, K. Kristiansen, Y. Golan, J. Israelachvili, Nat. Mater. 2008, 7, 527.

[45] S. Cherifi, R. Hertel, J. Kirschner, H. Wang, R. Belkhou, A. Locatelli, S. Heun, A. Pavlovska, E. Bauer, J. Appl. Phys. 2005, 98, 043901.

[46] F. Kronast, N. Friedenberger, K. Ollefs, S. Gliga, L. Tati-Bismaths, R. Thies, A. Ney, R. Weber, C. Hassel, F. M. Römer, others, Nano Lett. 2011.

[47] V. F. Puntes, P. Gorostiza, D. M. Aruguete, N. G. Bastus, A. P. Alivisatos, Nat. Mater. 2004, 3, 263.

[48] M. V. Rastei, S. Colis, J. P. Bucher, Chem. Phys. Lett. 2006, 417, 217.

[49] M. V. Rastei, S. Colis, R. Meckenstock, O. Ersen, J. P. Bucher, Surf. Sci. 2006, 600, 2178.

[50] H. Lichte, Ultramicroscopy 1996, 64, 79.

[51] R. E. Dunin-Borkowski, M. R. McCartney, D. J. Smith, S. S. P. Parkin, Ultramicroscopy 1998, 74, 61.

[52] H. Lichte, M. Lehmann, Rep. Prog. Phys. 2008, 71, 016102.

[53] A. Kakay, E. Westphal, R. Hertel, IEEE Trans. Magn. 2010, 46, 2303.

[54] R. Hertel, In Handbook of Magnetism and Advanced Magnetic Materials; John Wiley \& Sons, Ltd, 2007; pp. 1003-1020.

[55] L. D. Landau, E. M. Lifshitz, Phys. Z. Sowjetunion 1935, 8, 153.

[56] T. L. Gilbert, IEEE Trans. Magn. 2004, 40, 3443.

[57] W. F. Brown, Micromagnetics; Interscience Publishers, 1963.

[58] H. Kronmüller, M. Fähnle, Micromagnetism and the Microstructure of Ferromagnetic Solids; Cambridge University Press, 2003.

[59] A. Yamasaki, W. Wulfhekel, R. Hertel, S. Suga, J. Kirschner, Phys. Rev. Lett. 2003, 91.

[60] D. R. Fredkin, T. R. Koehler, IEEE Trans. Magn. 1990, 26, 415.

[61] S. Börm, L. Grasedyck, Computing 2004, 72, 325. 